\title{
Forschungsziele und Methode
}

Gegenstand dieser Untersuchung sind Werke, die das Resultat komplexer und vielfältiger Medienkombinationen, intermedialer Transfers und Transformationen sind oder an denen sich zahlreiche intra-, inter- und transmediale Bezüge erkennen lassen. ${ }^{1}$ Zunächst einmal ist Thomas Manns Doktor Faustus selbst ein sehr geeigneter literarischer Text zur Erforschung von Intermedialität, da er die Grenzen der Literatur mehrfach überschreitet und eine Annäherung an bildende Kunst und Musik wagt. ${ }^{2}$ Auch enthält diese Arbeit Analysen von Kompositionen und Werken, die verschiedene Medien, wie etwa Manzonis Doktor Faustus $^{3}$ oder Boehmers Apocalipsis cum figuris ${ }^{4}$ kombinieren und die auf Thomas Manns höchst kontroversen „Musiker-Roman“ (Ent: 25) reagieren, indem sie Mikroformen des Romans verschiedener Art (Motive, fiktive Werke, Figuren) in ein anderes kommunikativ-semiotisches System transferieren. ${ }^{5}$ Diese Werke, wie z. B. Hagens To Zeitblom ${ }^{6}$ oder Henzes Violinkonzert ${ }^{7}$ gehen dadurch ihrerseits an die Grenze zwischen den beiden Gebieten der Musik und der Literatur

\footnotetext{
${ }^{1}$ Zur hier verwendeten Terminologie, die im Folgenden erläutert wird, siehe u. a. Rajewsky, I. O.: Intermedialität. Tübingen/Basel: Francke 2002, S. 15-18 und Gess, Nicola: Intermedialität reconsidered. Vom Paragone bei Hoffmann bis zum Inneren Monolog bei Schnitzler. In: Poetica 40 (2010) H. 1-2, S. 139-168 (insb. S. 141-144).

${ }^{2} \mathrm{Vgl}$. Einleitung.

${ }^{3}$ Vgl. 5.2.1, 6.2, 7.2.3, 8.2.4, 10.2 u. 11.2.3.

${ }^{4} \mathrm{Vgl}$. 4.2.1.

${ }^{5}$ Vgl. Gess: Intermedialität reconsidered, S. 141. Gess stützt sich auf Paech, Joachim: Intermedialität. Mediales Differenzial und transformative Figurationen. In: Helbig, Jörg (Hrsg.): Intermedialität. Theorie und Praxis eines interdisziplinären Forschungsgebiets. Berlin: Schmidt 1998, S. 14-30 (insb. Kapitel 2).

${ }^{6} \mathrm{Vgl}$. 7.2.2.

${ }^{7}$ Vgl. 8.2.2, 9.2 u. 11.2.2.
} 
oder kombinieren beide kommunikativ-semiotischen Systeme in einem sekundären intermedialen und plurimedialen Produkt, wie z. B. Ránkis Leverkühns Abschied ${ }^{8}$ oder Lenners' Hetaera Esmeralda. ${ }^{9}$ Auch auf diese Untersuchung selbst lässt sich der Begriff der Grenzüberschreitung anwenden. Um grenzüberschreitende Werke analysieren zu können, muss nicht nur die Kunst, sondern auch die Wissenschaft selbst an die Grenzen des eigenen Faches gehen und mit anderen Disziplinen interagieren. Nur so kommt man zu plausiblen Ergebnissen: Die vorliegende, von einer literarischen Vorlage ausgehenden Studie integriert aus diesem Grund musikwissenschaftliche Kategorien und Werkzeuge in das eigene Analyseinstrumentarium. Beispielsweise geht es nicht lediglich darum zu zeigen, dass bestimmte Figuren des Romans in einer Komposition wiederzufinden sind, z. B. die Figur des Teufels samt ihren drei Gestalten wie im Roman in Manzonis Oper. ${ }^{10}$ Vielmehr ist es für die Analyse sehr fruchtbar zu untersuchen, mit welchen spezifischen Mitteln eines musikalischen Bühnenwerkes, etwa Kostüm, Bühnenbild, Stimmtypen, diese Figur realisiert wird und welche Implikationen diese Aspekte für den Kontext und das Medium bzw. die Medien der jeweiligen intermedialen Transposition haben (im Fall von Manzonis Oper: Mailand, 1989, Teatro alla Scala). Die Untersuchung der jeweiligen Rezeption des Romans geschieht somit nicht ausschließlich aus literaturwissenschaftlicher Perspektive und kann demnach die spezifischen Eigenschaften des neuen Mediums für umfassende Beobachtungen produktiv machen.

\section{$1.1 \quad$ Begriffsklärung}

Die Ausführungen der darauf folgenden Kapitel sind im intermedialen Bereich angesiedelt: Da unter die Kategorie ,Intermedialität" verschiedene Phänomene fallen und da sich dieses Forschungsparadigma durch eine ausdifferenzierte Vielfalt an Ansätzen, Definitionen und Methoden auszeichnet, ${ }^{11}$ erscheint eine kurze Besprechung der für die spätere Analyse verwendeten Begriffe und Kategorien unabdingbar.

\footnotetext{
${ }^{8} \mathrm{Vgl}$. 5.2.2.

${ }^{9} \mathrm{Vgl}$. 8.2.3.

${ }^{10} \mathrm{Vgl}$. 6.2.

${ }^{11}$ Siehe dazu Rajewsky: Intermedialität, S. 2-5.
} 


\subsubsection{Intermedialität}

Zunächst werden drei Definitionen von Intermedialität vorgestellt. Nach Irina Rajewsky umfasst Intermedialität „Mediengrenzen überschreitende Phänomene, die mindestens zwei konventionell als distinkt wahrgenommene Medien involvieren“. ${ }^{12}$ Werner Wolf greift im Metzler-Lexikon auf diese Definition zurück und differenziert zwischen einem weiten und einem engen Verständnis von Intermedialität: Im ersten Fall sei Intermedialität ,jedes Überschreiten von Grenzen zwischen konventionell als distinkt angesehenen Ausdrucks- oder Kommunikationsmedien“13 im zweiten Fall, also ,,in einem engeren, ,werkinternen“ Sinn analog zur Intertextualität, die eine in einem Text nachweisliche Einbeziehung mindestens eines weiteren (verbalen) Textes bezeichnet“, ${ }^{14}$ sei sie „eine in einem Artefakt nachweisliche Verwendung oder (referentielle) Einbeziehung wenigstens zweier Medien“. ${ }^{15}$ Uwe Wirth sieht Intermedialität als ,transdisziplinäre ,Schnittstelle " zwischen Literatur-, Kultur- und Medienwissenschaft" ${ }^{\text {" }}{ }^{16}$ wertet sie gar als zentrales Konzept ,für die Kopplung von Literaturwissenschaft und Medienkultur"17 und weist auf die Doppeldeutigkeit des Begriffs ,Schnittstelle“ hin, der ,gleichermaßen einen Moment der Grenzziehung und der Grenzüberschreitung "18 impliziere. Gemeinsam ist diesen Definitionen die Erwähnung des Begriffs ,Medium“ und nicht ,Text', was auf eine Verortung der Diskussion im intermedialen und nicht im intertextuellen Bereich im Sinne eines entgrenzten Textbegriffs schließen lässt. Darüber hinaus stellen alle drei Definitionen fest, dass Intermedialität ohne Einbeziehung oder Verwendung mindestens zweier Medien nicht möglich ist (sonst würde man von ,Intramedialität" oder ,Intertextualität' sprechen) und dass die Analyse intermedialer Phänomene eine inter- und transdisziplinäre Perspektive voraussetzt, die zu einem verschärften

\footnotetext{
${ }^{12}$ Ebd., S. 13.

${ }^{13}$ Wolf, W.: Intermedialität. In: Nünning, Ansgar (Hrsg.): Metzler Lexikon. Literatur und Kulturtheorie. Ansätze - Personen - Grundbegriffe. Stuttgart: Metzler 2013 [1998], 5. erw. und aktual. Aufl., S. 344 ff., hier: S. 344.

${ }^{14}$ Ebd.

${ }^{15}$ Ebd. Zur werkinternen und werkexternen Intermedialität siehe auch Wolf: Musik in Literatur: Showing, S. 98. Auf die beiden Begriffe wird später eingegangen.

${ }^{16}$ Wirth, Uwe: Hypertextuelle Aufpfropfung als Übergangsform zwischen Intermedialität und Transmedialität. In: Meyer, Urs, Roberto Simanowski u. Christoph Zeller (Hrsg.): Transmedialität. Zur Ästhetik paraliterarischer Verfahren. Göttingen: Wallstein 2006, S. 19-38, hier: S. 19.

${ }^{17}$ Ebd.

${ }^{18}$ Ebd., Herv. i. O.
} 
Blick über Möglichkeiten und Unmöglichkeiten der Grenzüberschreitung, also über mediale Kontinuitäten und Differenzen, führt.

\subsubsection{Medium}

Eine Definition von Intermedialität hängt vom jeweiligen Verständnis des Begriffs ,Medium‘ ab. In Anlehnung an Publikationen von Rajewsky und Wolf stützt sich die vorliegende Arbeit auf ein weites Konzept von Medium als ein so Werner Wolf - „konventionell im Sinn eines kognitiven frame of reference als distinkt angesehenes Kommunikationsdispositiv““. ${ }^{19}$ Der Vorteil dieses Verständnisses gegenüber einem, das Medium als ,technisch-materiell definierte[n] Übertragungskanal von Informationen“20 begreift, liegt darin, dass man sowohl Medien wie die Literatur, die sich in der Regel eines einzigen semiotischen Systems bedienen, der schriftlich fixierten Sprache, als auch andere, z. B. das im Rahmen dieser Untersuchung wichtige Medium der Oper, das auf mehrere semiotische Systeme (Bild, Musik, Text) zurückgreift, als ,,(Einzel-)Medien““21 bezeichnen kann. ${ }^{22}$

\subsubsection{Intertextualität/Intramedialität}

In seiner Definition von Intermedialität ,,in einem engeren, ,werkinternen ‘ Sinn“ ${ }^{\text {(23 }}$ deutet Werner Wolf bereits darauf hin, dass eine Analogie zwischen Intermedialität und Intertextualität besteht, und definiert dieses letzte Phänomen als „,in einem Text nachweisliche Einbeziehung mindestens eines weiteren (verbalen) Textes“. ${ }^{24}$ Geht man von einem weitgefassten Textbegriff im Sinne Kristevas aus, so wird der Text nicht nur als verbaler Text begriffen, sondern z. B. auch als

\footnotetext{
${ }^{19}$ Wolf, W.: Intermedialität. Ein weites Feld und eine Herausforderung für die Literaturwissenschaft (2002). In: Bernhart, Walter (Hrsg.): Selected Essays on Intermediality by Werner Wolf (1992-2014). Theory and Typology, Literature-Music Relations, Transmedial Narratology, Miscellaneous Transmedial Phenomena. Leiden/Boston: Brill Rodopi 2018, S. 63-91, hier: S. 65.

${ }^{20}$ Ebd.

${ }^{21}$ Rajewsky: Intermedialität, S. 7.

${ }^{22}$ Zum Begriff ,Medium“ siehe auch: Robert, Jörg: Einführung in die Intermedialität. Darmstadt: WBG 2014, S. 21 ff.

${ }^{23}$ Wolf: Intermedialität, S. 344.

${ }^{24}$ Ebd.
} 
bildlicher und musikalischer. Aus einer solchen Sicht heraus scheint der Begriff ,Intermedialität“ überflüssig zu sein, da die logische Konsequenz dieser ,radikalen Entgrenzung und Metaphorisierung des Textbegriffs“ 25 die „Entgrenzung des Intertextualitätsbegriffs“" 26 selbst wäre. ${ }^{27}$

Folglich würde man in dieser Studie beispielsweise lediglich vom Text reden, sowohl in Bezug auf die Musik als auch auf die Literatur. Der Rückgriff aber auf einen kommunikativ-semiotisch intendierten Begriff des Mediums und infolgedessen auf den Begriff der ,Intermedialität' ermöglicht in erster Linie die Beachtung medialer Differenzen, die in dieser Untersuchung zu einer umfassenden Rekonstruktion der jeweiligen Rezeption im neuen Medium beitragen. Wie Wirth in der bereits erwähnten Definition hervorhebt, subsumiert der Terminus ,Intermedialität‘ die beiden Gesten der Grenzüberschreitung und Grenzziehung; der letztgenannte Gestus besteht etwa in der Anerkennung einiger grundlegender Unterschiede in den Möglichkeiten der Einbeziehung eines weiteren verbal fixierten Textes in einem Roman, was im Gegensatz zur Einbeziehung einer musikalischen Komposition in einem Roman steht, z. B. in der „Erkenntnis, daß ein literarischer Text das [...] [musikalische] System nicht realisieren, sondern immer nur ,thematisieren", ,imitieren" oder ,evozieren" kann" ${ }^{28}$ Diese terminologische und systematische Grenzziehung ermöglicht eine fundierte Erfassung von Grenzüberschreitungen, welche die Potentialität der jeweiligen Medien, die einbezogen oder verwendet werden, stärker berücksichtigt. In Anlehnung an Rajewsky wird daher in der vorliegenden Studie der Begriff der Intertextualität durch den der Intramedialität weitgehend ersetzt. Dadurch soll verdeutlicht werden, dass die Analysen von dem vorher erläuterten kommunikativ-semiotischen Medium-Begriff ausgehen und als wichtige Voraussetzung die Frage ansehen, ob die betrachteten Phänomene innerhalb eines bestimmten Mediums oder zwischen zwei oder mehreren Medien, also zwei oder mehreren konventionell als unterschiedlich angesehenen kommunikativ-semiotischen Systemen, angesiedelt sind. ${ }^{29}$

\footnotetext{
${ }^{25}$ Rajewsky: Intermedialität, S. 48.

${ }^{26}$ Ebd.

${ }^{27}$ Vgl. auch Kristeva, Julia: Bakthine, le mot, le dialogue et le roman. In: Critique 13 (1967), S. 438-465.

${ }^{28}$ Rajewsky: Intermedialität, S. 57.

${ }^{29}$ Rajewsky benutzt den Terminus ,Intertextualität‘ nur als Subkategorie der Intramedialität, und zwar als alternative Bezeichnung der intramedialen Einzelreferenz. Siehe etwa ebd., S. 157. Zur Unterscheidung von Intertextualität zur Intermedialität vgl. auch Robert: Einführung in die Intermedialität, S. 20 f.
} 


\subsubsection{Transmedialität}

Eine weitere, nötige Präzisierung der hier verwendeten Begriffe betrifft die ,Transmedialität". Auch in diesem Fall wird auf Rajewskys Untersuchung rekurriert, die als wichtiges Kriterium zur Unterscheidung zwischen Intermedialität und Transmedialität das Gebundensein ,,an eine bestimmte Medienspezifik“30 bzw. „an eine bestimmte mediale Präsentationsform “31 ${ }^{\text {betrachtet. }}{ }^{32}$ Ihrer Auffassung nach bezeichnet Transmedialität ${ }^{33}$

medienunspezifische Phänomene, die in verschiedenen Medien mit den dem jeweiligen Medium eigenen Mitteln ausgetragen werden können, ohne daß hierbei die Annahme eines kontaktgebenden Ursprungsmediums wichtig oder möglich ist.

Beispiele für Transmedialität wären dementsprechend etwa die Parodie, die als Genre nicht nur dem Medium Literatur zugeordnet werden kann sowie christliche Motive, die als „Teil des allgemeinen Kulturguts [...] nicht (mehr) an eine bestimmte mediale Präsentationsform gebunden sind“. ${ }^{34}$ Wenn beispielsweise in Kapitel vier über die Apocalipsis cum figuris dargestellt wird, wie sich der apokalyptische Diskurs in Thomas Manns Roman und in den Kompositionen entfaltet, dann bewegt sich die Analyse auch auf dem Gebiet der Transmedialität. Dabei verlässt sie jedoch den Bereich der Intermedialität nicht, da die behandelten Musikwerke zugleich den apokalyptischen Diskurs von Doktor Faustus und den apokalyptischen Diskurs im Allgemeinen (teil-)reproduzieren. Das betrifft auch die Transposition von parodistischen Elementen des Romans, etwa in Searles Lamentation: ${ }^{35}$ Indem diese Medienkombination parodistische Elemente der fiktiven Kantate Leverkühns (teil-)reproduziert, setzt sie sich auch automatisch mit dem transmedialen Genre der Parodie auseinander und reproduziert und aktualisiert auch dieses in der Medienkombination. Aus diesen Beispielen geht hervor, dass die Grenzen zwischen Intermedialität und Transmedialität oft nicht scharf gezogen werden können. Besonders deutlich wird das in dieser Untersuchung,

\footnotetext{
${ }^{30}$ Rajewsky: Intermedialität, S. 73.

${ }^{31}$ Ebd.

${ }^{32}$ Andere Studien subsumieren unter ,Transmedialität‘ auch Phänomene des Medienwechsels sowie intermediale Bezugnahmen. Siehe ebd., S. 207.

${ }^{33}$ Ebd., S. 207.

${ }^{34}$ Ebd., S. 73.

${ }^{35} \mathrm{Vgl}$. 5.2.4.
} 
weil sie sich sowohl mit intermedialen Bezugnahmen als auch mit intermedialen Transpositionen $^{36}$ und Medienkombinationen beschäftigt. ${ }^{37}$

\subsubsection{Klassifikationen und Funktionen von Intermedialität}

Um die drei letztgenannten Begriffe zu erläutern, sei hier wieder auf Rajewsky verwiesen: Die Forscherin nimmt eine Untergliederung von Intermedialität in die drei Subkategorien der intermedialen Bezugnahme, des Medienwechsels und der Medienkombination vor. Die erste Subkategorie umfasse ${ }^{38}$

Verfahren der Bedeutungskonstitution eines medialen Produkts durch Bezugnahme auf ein Produkt (=Einzelreferenz) oder das semiotische System (=Systemreferenz) eines konventionell als distinkt wahrgenommenen Mediums mit den dem kontaktnehmenden Medium eigenen Mitteln.

Nur Letzteres zeichne sich durch seine materielle Präsenz aus. Beispiele aus der vorliegenden Untersuchung wären gemäß dieser Definition die Bezugnahme auf Beethovens Neunte Symphonie, auf musikalische Klageformen oder auf die Zwölftontechnik. Die Subkategorie des Medienwechsels hat Rajewsky zufolge mit der ,Transformation eines medienspezifisch fixierten Produkts bzw. ProduktSubstrats in ein anderes, konventionell als distinkt wahrgenommenes Medium“39 $\mathrm{zu}$ tun, auch in diesem Fall ist ,nur letzteres [...] materiell präsent“"40 Als

\footnotetext{
${ }^{36}$ Zum Begriff siehe Fußnote 40.

${ }^{37}$ Siehe auch ebd., S. 104 f.

${ }^{38}$ Ebd., S. 19.

${ }^{39}$ Ebd.

${ }^{40}$ Ebd. Gess kritisiert den Begriff ,Medienwechsel ' und schlägt, insbesondere im Kontext musikliterarischer Intermedialität, den Begriff ,Medientransformation“ vor (zu beachten ist aber, dass sich hier Rajewsky beim Beschreiben des Phänomens bereits des Terminus bedient): „Es geht bei musik-literarischer Intermedialität nicht um den Wechsel eines medienunabhängigen etwas von einem Medium ins andere, sondern um die Transformation des Mediums selbst im Sinne der Transformation medienspezifischer Eigenschaften und Verfahren". Die Annahme eines Wechselns sei in diesem Fall besonders problematisch, speziell im Fall des „Übergang[s] von Musik in literarischen Text“ (z. B. Kontrapunkt in einem Text), ,weil Musik keine medienunabhängigen, inhaltliche Substrate zugewiesen werden können, die man aus ihr herauslösen und in ein anderes Medium wechseln lassen könnte“. Gess: Intermedialität reconsidered, S. 142, Herv. i. O. Vielleicht ist diesbezüglich Wolfs Terminus der, intermedialen Transposition` zu präferieren, insofern er beide Gesten des Transfers und der medienspezifischen Transformation in den Fokus rückt. Siehe Wolf, W.: Intermedialität. Ein weites Feld und eine Herausforderung, S. 77 u. Ders.:
} 
Medienwechsel lassen sich vor allem aufgrund des Bezugs auf Thomas Manns Roman, der oft durch die Paratexte programmatisch deklariert wird, die Mehrheit der hier betrachteten Kompositionen einstufen: Henzes Violinkonzert trägt z. B. den Untertitel Drei Porträts aus dem Roman „Dr. Faustus“ von Thomas Mann. ${ }^{41}$ Eine Ausnahme stellen etwa die Kompositionen von Hagen, ${ }^{42}$ Ruzicka ${ }^{43}$ und Kurz ${ }^{44}$ dar, von denen es angebrachter wäre zu sagen, dass sie intermediale Bezugnahmen auf Doktor Faustus enthalten, da sehr kleine Mikroformen aus dem Roman das Medium ,wechseln“ und es sich daher eher um Bezugnahmen bzw. sehr kleine Einbeziehungen handelt. Die dritte Subkategorie, der sich fast alle Kompositionen dieser Studie zuordnen lassen (mit Ausnahme von Henzes Violinkonzert, Ruzickas ,...ZURÜCKNEHMEN...“ und Fines Four pieces from „Doktor Faustus “), ${ }^{45}$ sind Medienkombinationen. ${ }^{46}$ Rajewsky definiert sie als ,die Kombination bzw. das Resultat der Kombination mindestens zweier, konventionell als distinkt wahrgenommener Medien“ ${ }^{47}$ die ,,in ihrer Materialität präsent sind und jeweils auf ihre eigene, medienspezifische Weise zur (Bedeutungs-)Konstitution des Gesamtprodukts beitragen“". ${ }^{48}$ Das wahrscheinlich beste Beispiel ist die Oper, welche - auf der reinen Ebene der Lektüre, d. h. nur anhand der Partitur - die Medien des Textes und der Musik kombiniert (zieht man die Aufführung in Betracht, so erweitert sich die Anzahl an Medien).

Des Weiteren werden bei Rajewsky intermediale Bezüge in die zwei Hauptkategorien der Einzelreferenz und Systemreferenz untergliedert. ${ }^{49} \mathrm{Im}$ ersten Fall wird auf ein Produkt eines anderen Mediums Bezug genommen, in Doktor Faustus z. B. auf Monteverdis Lamento d'Arianna bei der Beschreibung der Weheklag. Diese bloße Thematisierung von Monteverdis musikalischem Werk könnte eine Systemreferenz indizieren, also beispielsweise die Simulation oder

Narrativity in Instrumental Music? A Prototypical Narratological Approach to a Vexed Question (2008). In: Bernhart (Hrsg.): Selected Essays on Intermediality by Werner Wolf, S. $480-500$.

${ }^{41} \mathrm{Vgl}$. 8.2.2.

${ }^{42} \mathrm{Vgl}$. 7.2.2.

${ }^{43} \mathrm{Vgl}$. 5.2.3.

${ }^{44} \mathrm{Vgl}$. 4.2.2.

${ }^{45}$ Vgl. 7.2.1, 8.2.1 u. 11.2.1.

${ }^{46}$ Bei W. Wolf bilden ,Medienkombinationen“ zusammen mit ,Medienmischungen“ Unterkategorien von ,Plurimedialität‘. Siehe Wolf., W.: Intermedialität. Ein weites Feld und eine Herausforderung, ebd.

${ }^{47}$ Rajewsky: Intermedialität, S. 15.

${ }^{48}$ Ebd.

${ }^{49}$ Siehe etwa ebd., S. 157. 
(Teil-)reproduktion musikalischer Klageformen im Roman. ${ }^{50}$ Erster Schritt einer Intermedialitätsanalyse ist die Suche nach Markierungen im Text: ${ }^{51}$ Die vorliegende Studie folgt eben diesem Prozedere, das sich in der analytischen Praxis strukturiert und systematisch umsetzen lässt. In Bezug auf Doktor Faustus lässt sich etwa feststellen, dass Monteverdis Komposition nicht einfach erwähnt wird, sondern auch eine Systemreferenz auf musikalische Klageformen indiziert. Dies bestätigt nicht nur die Einzelreferenz auf Monteverdis Werk, sondern auch die explizite Systemerwähnung eben dieser Formen, sowohl in Monteverdis Schaffen als auch in der gesamten musikalischen Tradition (nicht nur) des Abendlandes. ${ }^{52}$ Die Systemerwähnung stellt bei Rajewsky eine Subkategorie der Oberkategorie der Systemreferenz dar: Ein semiotisches System kann einfach explizit erwähnt werden (siehe z. B. Zeitbloms explizite Erwähnung ,ich spiele die Viola d'amore“, DF: 12) ${ }^{53}$ oder eine wichtige Voraussetzung für die Weiterführung der Analyse sein. Jene Markierung kann sich als Indiz für eine ,fremdmedial bezogene Illusionsbildung“ 54 also für eine „Systemerwähnung qua Transposition“55 erweisen; dementsprechend könnte man sich fragen, ob das System, im Fall des vorher genannten Beispiels das musikalische System des Spielens der Viola d'amore, im Text auch evoziert oder simuliert wird. Wird im Text eine „Ähnlichkeitsbeziehung zwischen dem ,Vergleichs'objekt und bestimmten Komponenten des [...] [musikalischen] (Sub-)Systems hergestellt" 56 die ,,in die Bedeutungskonstitution des jeweiligen Textes“57 eingehen? Kommt es vielleicht sogar $\mathrm{zu}$ „einer sprachlichen Imitation der [...] [musikalischen] Mikroform“, ${ }^{8}$ also zu einer Ähnlichkeitsbeziehung, die ,nicht nur ,über das Bezugssystem redend“ suggeriert oder wie in anderen Fällen konstatiert, sondern tatsächlich diskursiv hergestellt wird" "? ${ }^{59}$ Beantwortet man die erste oder die zweite Frage positiv, so hat man es nicht nur mit einer expliziten Systemerwähnung, sondern zusätzlich auch jeweils mit einer evozierenden und einer simulierenden Systemerwähnung zu tun.

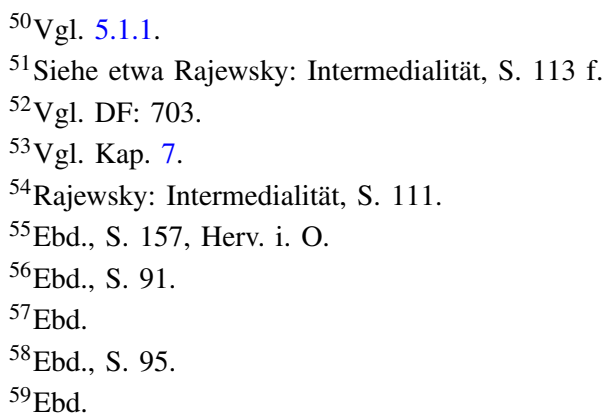


Die dritte Unterform der Systemerwähnung ist die ,(teil-)reproduzierende Systemerwähnung“, ${ }^{60}$ die jedoch nicht auf das genannte Beispiel angewendet werden kann, da sich das Spielen der Viola d'amore weder als medienunspezifisch noch als medial deckungsgleich begreifen lässt. (Teil-)reproduzieren lassen sich in einem anderen Medium nur medienunspezifische Elemente eines bestimmten kontaktgebenden Systems, beispielsweise „charakteristische inhaltliche Elemente, Figuren, Figurenkonstellationen, Dialog- und/oder Handlungsstrukturen “61 eines Genres: Der transmediale apokalyptische Diskurs kann etwa in den Kompositionen, also im Medium der Musik, wohl reproduziert werden, der apokalyptische Diskurs von Doktor Faustus kann hingegen mit den Mitteln der Musik nur partiell wiedergegeben werden, und zwar ,in seinen medienunspezifischen Bestandteilen". ${ }^{62}$ Medial deckungsgleiche Elemente bzw. Strukturen, die ebenfalls in einem anderen semiotischen System teilreproduziert werden können, sind etwa verbalsprachliche Komponenten: Um erneut auf Zeitbloms Beschreibung der Weheklag zu verweisen, lässt sich beispielsweise feststellen, dass zwar bestimmte sprachliche Elemente von Stabat Mater-Texten auch im Roman reproduziert werden können, sich aber die Makroform des musikalischen Stabat Mater nur durch ein Hinzudenken der Leser*innenschaft gänzlich wiedergeben lässt, folglich nicht mit den Mitteln des fiktionalen Textes. ${ }^{63}$ Dies stellt einen wichtigen Unterschied zur Intramedialität dar: Bei dieser ist hingegen auch eine Systemaktualisierung möglich, ${ }^{64}$ d. h. ein literarischer Text kann etwa bestimmte „literarische Subsysteme (spezifische Genres, Texttypen usw.)“65 erwähnen, reproduzieren und somit aktualisieren.

Häufen sich die intermedialen Systemerwähnungen in einem Text und tauchen diese dementsprechend nicht nur in einer Passage auf, so kann es Rajewsky zufolge zu einer Systemkontamination, also zu einer „Modifikation des kontaktnehmenden in Richtung auf das kontaktgebende System" ${ }^{\prime 66}$ kommen. Auch hier ist die Frage, ob eine fremdmedial bezogene Illusionsbildung hervorgerufen wird, zentral: Eine Bejahung führt zum nächsten Schritt der Analyse, nämlich

\footnotetext{
${ }^{60}$ Ebd., S. 157.

${ }^{61}$ Ebd., S. 104.

${ }^{62}$ Ebd., S. 108; vgl. Kap. 4.

${ }^{63} \mathrm{Vgl}$. 5.1.1.

${ }^{64}$ Abgesehen davon, weist Rajewsky darauf hin, ,,daß ein literarischer Text stets und per definitionem das System ,Literatur“ aktualisiert“. Ebd., S. 71, Herv. i. O.

${ }^{65}$ Ebd., S. 71.

${ }^{66}$ Ebd., S. 133.
} 
zur Unterscheidung zwischen einer „Systemkontamination qua Translation“67 und einer ,teilaktualisierende[n] Systemkontamination“. 68 Wichtiges Kriterium ist in diesem Fall die Feststellung der Art dieser ,kontaminierenden“ Elemente. ${ }^{69}$ Hat man es mit medienunspezifischen bzw. medial deckungsgleichen Elementen zu tun, so handelt es sich um eine teilaktualisierende Systemkontamination: Das fremdmediale System wird dadurch partiell zur Texterzeugung verwendet und folglich auch partiell aktualisiert. ${ }^{70}$ Es konnte z. B. in dieser Studie gezeigt werden, dass sich etwa medial deckungsgleiche Mikroformen nicht nur in der Beschreibung der Weheklag, sondern nahezu im gesamten Roman auffinden lassen, die auf das musikalische System, und speziell auf das der musikalischen Klagen verweisen, und dass auf diese Weise eine fremdmedial bezogene Illusionsbildung im Text hervorgerufen wird. Dies sprengt den Rahmen einer intermedialen (Teil-)reproduktion und ist als teilaktualisierende Systemkontamination zu werten. Wenn durchgehend medial spezifische Elemente auftreten und eine altermedial bezogenen Illusionsbildung in einem Text hervorgerufen wird, lässt sich dieses Phänomen hingegen der Subkategorie der Systemkontamination qua Translation zuordnen: Darunter versteht Rajewsky die „Übertragung oder Verschiebung fremdmedialer Regeln und Prinzipien auf oder in das literarische Medium“. ${ }^{71}$ Konstituiert sich der gesamte Text so, dass Regeln und Prinzipien übertragen werden, die dem literarischen Medium nicht inhärent sind, z. B. Regeln aus der Aufführungspraxis der Viola d'amore auf das Verfassen einer fiktionalen Schrift, so sprengt das ebenfalls den Rahmen einer Systemerwähnung und tritt in das Feld der Systemkontamination qua Translation ein.

Uwe Wirth entwirft ein Stufenmodell von Intermedialität, das zum Teil Rajewskys Phänomenbereiche der intermedialen Bezugnahme, des Medienwechsels und der Medienkombination sowie die Arten der intermedialen Bezüge (Einzelreferenz, Systemerwähnung, Systemkontamination) integriert. Die Nullstufe der Intermedialität bestehe im bloßen ,Thematisieren eines Mediums in einem anderen Medium, etwa [in] eine[r] literarische[n] Reflexion über die Malerei“" ${ }^{2}$

\footnotetext{
${ }^{67}$ Ebd., S. 145, Herv. i. O.

${ }^{68}$ Ebd.

${ }^{69}$ Obwohl Rajewsky betont, dass der Begriff ,Kontamination“ hier nicht in einem negativ konnotierten Sinne verwendet werde, scheint es schwierig, ihn im Sinne einer Befruchtung medialer Systeme zu verstehen. Siehe ebd., S. 133. Zur Metaphorizität intermedialer Begriffe sei hier auf Robert: Einführung in die Intermedialität, S. 24 verwiesen.

${ }^{70}$ Siehe Rajewsky: Intermedialität, S. $142 \mathrm{f}$.

${ }^{71}$ Ebd., S. 134.

${ }^{72}$ Wirth: Hypertextuelle Aufpfropfung, S. 32.
} 
was mit Rajewskys expliziter Systemerwähnung und Werner Wolfs intermedialem telling korrespondiert und ebenfalls ein Indiz für „,implizite Inszenierungen von Intermedialität" ${ }^{73}$ also für Evokation, Simulation und (Teil-)reproduktion nach Rajewsky oder intermediales showing laut Wolf, sein kann. ${ }^{74}$ Die erste Stufe definiert Wirth als die ,mediale Modulation der Konfiguration eines Zeichenverbundes“; 75 sie betreffe etwa den „Übergang von gesprochener Sprache in geschriebene": ${ }^{76}$ Solche Phänomene seien, so Wirth, erst dann als intermedial zu werten, ,wenn sie zu einer Re-Konfiguration des Zeichenverbundsystems führen“, ${ }^{77}$ z. B. im Fall einiger Text-Transfers wie der ,Transformation von dramatischen Texten in theatrale Aufführungen“. ${ }^{78}$ Ein Beispiel aus dieser Studie wäre etwa die Transformation von Manzonis Partitur in die Uraufführung im Teatro alla Scala: Der Vergleich zwischen gedruckter Partitur und Aufführung in den entsprechenden Abschnitten dieser Arbeit ließe sich demnach der ersten Stufe der Intermedialität nach Wirth zuordnen. Bei der zweiten Stufe der Intermedialität geht es um „die Kopplung verschieden konfigurierter Zeichenverbundsysteme etwa die Kopplung von Text und Bild“ ${ }^{79}$ Wirth erläutert die Besonderheit dieser Kopplung wie folgt: ${ }^{80}$

Im Gegensatz zu einem multimedialen Nebeneinander ist das intermediale Miteinander durch eine integrierende konzeptionelle und mediale Re-Konfiguration ausgezeichnet. Eine derartige Re-Konfiguration impliziert eine technisch-mediale und eine inszenierend-konzeptionelle Modulation der performativen Rahmenbedingungen. Das Modell hierfür sind mediale Hybridbildung und mediale Aufpfropfung.

Es handelt sich der Beschreibung entsprechend um eine Medienkombination, die Konsequenzen für die performativen Rahmenbedingungen hat: So z. B. in Hagens Komposition, die durch die in der Partitur vorgesehene Moderation inmitten der Performance die sonst rein instrumentale Komposition re-konfiguriert.

\footnotetext{
${ }^{73}$ Ebd.

${ }^{74}$ Zum intermedialen showing und telling siehe Wolf: Musik in Literatur: Showing, S. $95 \mathrm{f}$. und Ders: „The musicalization of fiction“. Versuche intermedialer Grenzüberschreitung zwischen Musik und Literatur im englischen Erzählen des 19. und 20. Jahrhunderts. In: Helbig (Hrsg.): Intermedialität, S. 133-164, hier: S. 133.

${ }^{75}$ Wirth: Hypertextuelle Aufpfropfung, S. 32, Herv. i. O.

${ }^{76}$ Ebd.

${ }^{77}$ Ebd.

${ }^{78}$ Ebd.

${ }^{79}$ Ebd.

${ }^{80}$ Ebd., Herv. i. O.
} 
Die dritte Stufe der Intermedialität ist Wirth zufolge die der ,konzeptionelle[n] Aufpfropfung“ 81 bei der ,das Konzept der medialen Konfiguration eines Zeichenverbundsystems auf ein anderes“ ${ }^{82}$ übertragen wird: Die konzeptionelle Aufpfropfung lasse sich folglich als ,Metapher für eine mediale Aufpfropfung “83 begreifen. Die Übertragung von Aufführungstechniken historischer Instrumente auf die Narration, etwa die der Scordatura, würde man demnach in diese Kategorie einordnen. ${ }^{84}$

Darüber hinaus unterscheidet Wirth zwischen einer von ihm sogenannten ,harten' und einer, weichen“ Intermedialität. Die ,harte‘ Intermedialität sei der Stufe zwei zuzuordnen, sprich: der medialen Hybridbildung und der medialen Aufpfropfung; ihre Untersuchung benötige ,ein technisches Wissen über die medialen Differenzen der gekoppelten Medien“" 85 das die ,weiche "Intermedialität, welche die Stufen eins und drei betrifft, nicht voraussetze. Bei ihr reiche ,ein konzeptionelles Wissen um mediale Differenzen" ${ }^{86}$ aus. Infolgedessen sei die ,weiche“ Intermedialität der Gegenstandsbereich einer ,,literaturzentrierten“ intermedialen Forschungsperspektive“ 87 im Sinne Werner Wolfs. „Paradoxerweise“" 88 schreibt jedoch Uwe Wirth, ${ }^{89}$

ist es nun ausgerechnet die harte Intermedialität der Stufe zwei, die dem Paradigma der Transmedialität am nächsten kommt, denn mediale Aufpfropfungen können nicht nur als Produkt einer intermedialen Kopplung, sondern auch als transmedialer Prozess des Übergangs in den Blick genommen werden: ein Prozess, in dessen Vollzug die gekoppelten Elemente ihre Identität gerade durch den Bezug zum anderen Medion, aber eben auch in Absetzung von diesem erwerben.

\footnotetext{
${ }^{81}$ Ebd., Herv. i. O.

${ }^{82}$ Ebd., S. 33.

${ }^{83}$ Ebd., Herv. i. O.

${ }^{84} \mathrm{Vgl}$. 7.1.2.

${ }^{85} \mathrm{Ebd}$.

${ }^{86}$ Ebd., S. 33.

${ }^{87}$ Ebd. Wirth bezieht sich hier auf die folgende Publikation Werner Wolfs: Wolf, W.: Intermedialität als neues Paradigma der Literaturwissenschaft? Plädoyer für eine literaturzentrierte Erforschung von Grenzüberschreitungen zwischen Wortkunst und anderen Medien am Beispiel von Virginia Woolfs „The String Quartet“ (1996). In: Bernhart (Hrsg.): Selected Essays on Intermediality by Werner Wolf, S. 3-37.

${ }^{88}$ Ebd., S. 33 f.

${ }^{89}$ Ebd.
} 
Während Rajewskys Kategorien eine gute analytische Basis zur Erforschung der „werk-/aufführungsintern nachweisbare[n]“90 Intermedialität bieten, betont Wirth in dem oben erwähnten Zitat die Notwendigkeit eines doppelten Blicks im Falle einer sogenannten ,medialen Aufpfropfung'. Diese könne (und sollte) gleichzeitig als Produkt und als Prozess in den Blick genommen werden, was ein Sich-Hinund-Her-Bewegen zwischen Intermedialität und Transmedialität zur Folge habe. Auch Wolf spricht von einer ,werk-/aufführungsübergreifend erschließbare[n]“91 Intermedialität, die ,nicht in einem Einzelwerk [...] ersichtlich“92 wird, ,sondern erst aus dem Vergleich eines Werkes mit altermedialen anderen“93 und die er in die Unterkategorien der, intermedialen Transposition', ein Beispiel wäre die ,Veroperung' eines Romans wie Manzonis Doktor Faustus, und der Transmedialität untergliedert. ${ }^{94}$ Da sich die vorliegende Studie mit werkinterner und werkexterner Intermedialität beschäftigt sowie mit zahlreichen medialen Aufpfropfungen bzw. intermedialen Transpositionen, bedarf die Analyse stets eines doppelten, manchmal simultanen Blicks auf das Produkt und den Prozess und verlässt, wenn nötig, das Gebiet der Intermedialität, um mit dem der Transmedialität zu interagieren. ${ }^{95}$

Weitere Kategorien aus der Intermedialitätsforschung, mit denen die Analysen der vorliegenden Studie arbeiten und nach denen sich Intermedialität differenzieren lässt, werden im Folgenden näher erläutert. Dass sich diese Arbeit vor allem einer musikliterarischen Intermedialität zuordnet, ist bereits deutlich geworden. Die „beteiligten Medien“96 der in dieser Studie behandelten intermedialen Produkte und Prozesse betreffen stets die Bereiche von Text und Musik, mit Ausnahme von Searles, Lenners und Beyers ${ }^{97}$ Kompositionen, die jeweils auch mit Rundfunk, Kunst und Fernsehen zu tun haben: Da es sich hier nicht nur um eine ,weiche' Intermedialität nach Wirth handelt, sondern auch um eine ,harte', die dementsprechend von profundem technischen Wissen nicht absehen kann, werden vor allem musik- und textbezogene Aspekte solcher plurimedialen Artefakte in den Blick genommen.

\footnotetext{
${ }^{90}$ Wolf: Musik in Literatur: Showing, S. 98.

${ }^{91}$ Ebd.

${ }^{92}$ Ebd., S. 96.

${ }^{93}$ Ebd.

${ }^{94}$ Siehe ebd, S. 98.

${ }^{95}$ Jedenfalls muss hier angemerkt werden, dass Intermedialität per definitionem ohne werkexterne Verweise kaum nachweisbar ist: Wie lässt sich etwa die Simulation einer Sonatenform in einem Roman zeigen, wenn nicht auf Beispiele aus der Musikgeschichte verwiesen wird?

${ }^{96}$ Wolf, W.: Intermedialität, S. 345.

${ }^{97} \mathrm{Vgl}$. 3.3.1.
} 
Sofern eine medienspezifische Dominanzbildung betrachtet wird, lassen sich die Kompositionen dieser Studie beiden Typologien, d. h. dem Typ „,mit Dominanz eines Mediums gegenüber einem oder mehreren anderen“98 (z. B.: Fine, Hagen und Henze) als auch dem ,ohne klare Dominanz"99 zuordnen (z. B.: Manzoni, Ránki und Searle): Es sind vor allem Medienkombinationen, bei denen sich kein klares Dominanzverhältnis feststellen lässt. Rückt ,die Quantität der intermedialen Bezugnahmen" ${ }^{100}$ in den Fokus der Klassifizierung, so lässt sich vorab festhalten, dass die hier präsentierten Kompositionen alle partiell sind, d. h. sie spiegeln nie das ganze Werk, sondern nur Teile davon wider. Auch bei Manzoni signalisiert das Wort scene im Untertitel der Oper, dass eine Auswahl getroffen wurde. Nur auf einer sehr theoretischen Ebene wäre es aufgrund der Textlänge möglich, einen Roman wie Doktor Faustus vollständig intermedial zu transponieren, was zu metamedialen Reflexionen ${ }^{101}$ über die Erzählzeiten der Literatur und die Aufführungszeiten der Musik führt.

Die Intermedialität lässt sich auch nach ihrer Genese klassifizieren. Im Fall des Romans selbst ist sie primär, also ,,von Anfang an Teil des Werkkonzeptes“, 102 wie man etwa Manns Tagebucheinträgen oder der Entstehung des „Doktor Faustus “ entnehmen kann. ${ }^{103}$ Da sich unter diesem Aspekt die hier behandelten Kompositionen mit Romanverfilmungen gleichsetzen lassen, hat man es mit sekundärer Intermedialität zu tun, insofern alle Werke ,erst im Nachhinein, [...] von fremder Hand"104 entstanden sind. Diese Beobachtung beinhaltet einige für die Analyse wichtige Implikationen: Die Kompositionen dieser Studie entstehen aus unterschiedlichen geographischen und historischen Prämissen, die u. a. für die im Werk sichtbare Rezeption des Romans eine Rolle spielen. Des Weiteren gilt es, die „Qualität des intermedialen Bezuges“105 in Betracht zu ziehen: Wie das erfolgen kann, wird im Folgenden erläutert. Diese von Wolf vorgeschlagene Kategorisierung schließt sich an die vorher erwähnte Unterscheidung

\footnotetext{
${ }^{98}$ Ebd.

${ }^{99}$ Ebd.

${ }^{100} \mathrm{Ebd}$.

${ }^{101}$ Zum Begriff,Metamedialität‘ vgl. Rajewsky: Intermedialität, S. 81.

${ }^{102}$ Wolf: Intermedialität, ebd.

${ }^{103}$ Vgl. etwa Ent: 13.

${ }^{104}$ Wolf: Intermedialität, ebd.

${ }^{105}$ Ebd.
} 
nach den beteiligten Medien an. Liegt ,direct or ,overt“ intermediality“, 106 also „manifeste“107 bzw. „offene“108 Intermedialität vor, so enthält die Komposition mehr als ein Medium, die dann ,in principle separately ,quotable“" sind, z. B. Musik und Text. ${ }^{109}$ Das Werk bzw. die Aufführung zeichnet sich folglich durch mediale Heterogenität, also durch die Ko-Präsenz mehrerer Medien aus. ${ }^{110}$ Wenn man hingegen von „verdeckte[r]“111 Intermedialität spricht, bleibt „die Oberfläche des betreffenden Werk-/Aufführungsteils medial homogen“, ${ }^{112}$ was zur Folge hat, dass sich die Analyse aufgrund der schwierigen Nachweisbarkeit von Intermedialität als problematischer erweist. Dies wird in der musikliterarischen Intermedialität bei der Betrachtung von Instrumentalmusik von der unter zeichentheoretischen Gesichtspunkten erschließbaren selbstreferentiellen Qualität der Musik erschwert, was dazu führt, dass sich in der Instrumentalmusik in der Regel kein intermediales telling, sondern nur showing identifizieren lässt. ${ }^{113}$ Ruzickas „,..ZURÜCKNEHMEN...“, Henzes Violinkonzert und Fines Four pieces zählen zu den wenigen verdeckten intermedialen Phänomenen dieser Studie, bei denen u. a. paratextuelle Angaben zur Nachweisbarkeit der Intermedialität beitragen und doch auch Verweise auf Außermusikalisches in einer instrumentalen Form ermöglichen. ${ }^{114}$

Die vorliegende Studie beschränkt sich jedoch nicht ausschließlich auf die Erfassung und Beschreibung intermedialer und transmedialer Phänomene im Roman und in den Kompositionen, sondern sie geht auch der Frage nach, welche Funktionen die indizierten Strategien, die eine doppelte Perspektive, d. h. werkintern und werkextern, folglich auf Produkt und Prozess erforderlich machen,

\footnotetext{
${ }^{106}$ Wolf, W.: Musicalized Fiction and Intermediality: Theoretical Aspects of Word and Music Studies (1999). In: Bernhart (Hrsg.): Selected Essays on Intermediality by Werner Wolf, S. 238-258, hier: S. 243.

${ }^{107}$ Wolf: Intermedialität, S. 345.

${ }^{108}$ Wolf: Musik in Literatur: Showing, S. 98.

${ }^{109}$ Wolf: Musicalized Fiction, S. 243.

${ }^{110}$ Wolf: Musik in Literatur: Showing, S. 98.

${ }^{111}$ Ebd.

${ }^{112}$ Ebd.

${ }^{113}$ Siehe ebd., S. 97; Calzoni, Raul, Peter Kofler u. Valentina Savietto: Einleitung. In: Dies. (Hrsg.): Intermedialität - Multimedialität, S. 9-19, hier: S. 12 f.; Adorno, Theodor W.: Fragment über Musik und Sprache (1956/57). In: Knaus, Jakob (Hrsg.): Sprache, Dichtung, Musik. Texte zu ihrem gegenseitigen Verständnis von Richard Wagner bis Theodor W. Adorno. Tübingen: Niemeyer 1973, S. 71-74; Eco, Umberto: Einführung in die Semiotik. Übers. v. Jürgen Trabant. München: Fink, S. 104-107.

${ }^{114}$ Siehe Wolf: Narrativity in Instrumental Music?, S. 492.
} 
übernehmen. ${ }^{115}$ Mediale Kontinuitäten und Differenzen rücken somit in den Fokus: Es geht nicht nur darum, was in das Musikwerk transponiert wird, sondern auch darum, wie das geschieht. Dadurch wird man zwangsläufig mit der Frage konfrontiert, ob sich dies nur als Resultat einer bestimmten Rezeptionsweise erklären lässt ${ }^{116}$ oder vielleicht der Schwierigkeit entspringt, im Medium der Musik oder z. B. im plurimedialen Format der Oper oder des Monodramas bestimmte Mikroformen des Romans zu reproduzieren.

Als letzter Punkt dieses Unterabschnitts lohnt es sich, nun der Frage nachzugehen, wie diese Effekte und Funktionen von Intermedialität benannt und dementsprechend eingeordnet werden können. Gess schlägt bezüglich der Funktion von Musik im Verhältnis zum Text im Kontext von Medienkombinationen einige Kategorien, die für die Analyse eines einzigen Produkts gedacht sind, vor: „Verstärkung, Kommentierung“ 117 „Infragestellung“, 118 „Verfremdung“119 und Ergänzung. ${ }^{120}$ Des Weiteren weist die Forscherin darauf hin, dass die Differenz zwischen Text und Musik ,minimiert“121 oder ,gerade herausgestellt und konturiert werden“122 kann. Diese Arbeit bedient sich des Vokabulars von Gess” Aufsatz, das nicht nur für die Betrachtung eines einzigen intermedialen Produktes wie im genannten Text, sondern in der vorliegenden Untersuchung auch für die Analyse zweier intermedialen Produkte verwendet wird (sozusagen eines *Genoproduktes und eines *Phänoproduktes): In welchem Verhältnis steht etwa die Apocalipsis cum figuris von Boehmer zu Thomas Manns Roman? Beide Produkte, Vorlage und Komposition, werden in die Analyse einbezogen. Im Laufe der Studie werden zudem neue Benennungen für die Untersuchung intermedialer Effekte vorgeschlagen: So ist etwa von Revision zu sprechen, wenn eine Mikroform aus dem Roman nicht nur in Frage gestellt, sondern auch einer Revision unterzogen wird. Beispielsweise revidieren einige Kompositionen Leverkühns Absicht

\footnotetext{
${ }^{115}$ Bereits Rajewsky betont, die intermediale Analyse solle sich nicht nur auf eine Kategorisierung beschränken, sondern auch die Funktionen intermedialer Phänomene mit einbeziehen. Vgl. etwa Rajewsky: Intermedialität, S. 111.

${ }^{116}$ Siehe diesbezüglich die in Anlehnung an Kramer und Georgiades von Arne Stollberg vorgestellten semantischen Typologien der Textvertonung: Stollberg, Arne: Kombination von Literatur und Musik. In: Gess u. Honold (Hrsg.): Handbuch Literatur \& Musik, S. 5777, hier: S. 64.

${ }^{117}$ Gess: Intermedialität reconsidered, S. 152.

${ }^{118} \mathrm{Ebd}$.

${ }^{119}$ Ebd., S. 156.

${ }^{120}$ Vgl. ebd., S. 141.

${ }^{121}$ Ebd., S. 158.

${ }^{122}$ Ebd.
} 
einer Zurücknahme der Neunten Symphonie, indem sie Zitate aus Beethovens letztem symphonischen Werk in das eigene Material integrieren. Somit stellen sie die Unmöglichkeit von Leverkühns Versuch heraus. Schließlich kann durch die Musik der Text „hörbar“ werden, was beispielsweise bei Searle deutlich wird, der sich zum Ziel setzt, Zeitbloms Beschreibungen von Leverkühns Umsetzung der Zwölftontechnik erklingen zu lassen.

\subsubsection{Narrativität in der instrumentalen Musik?}

Nicht selten interagiert zudem die Analyse mit Kategorien im Sinne einer transmedialen und intermedialen Narratologie und wirft die Frage auf, wie etwa bestimmte Motive des Romans in der Vertonung (teil-)reproduziert werden oder ob sich narrative Strategien der Vorlage in der Komposition wiederfinden lassen und wie es überhaupt möglich ist, beispielsweise Unzuverlässigkeit im Medium der Musik darzustellen. ${ }^{23}$ Die Untersuchungsperspektive ist auch in diesem Fall werkintern und werkextern, transmedial und intermedial und kombiniert das Instrumentarium der Intermedialitätsforschung mit dem der Narratologie. Insofern entspringt diese Studie dem doppelten Wunsch, eine Forschungslücke in der sonst stark ausdifferenzierten Forschungsliteratur zu Thomas Manns Doktor Faustus zu füllen und Rajewskys Aufforderung nachzukommen, intermediale Kategorien auf immer unterschiedliche Artefakte anzuwenden, um sie dadurch zu erproben, zu erweitern und vielleicht auch zu revidieren. ${ }^{124}$

An dieser Stelle scheint eine methodische und begriffliche Präzisierung bezüglich der Positionierung dieser Untersuchung der transmedialen Narratologie gegenüber sinnvoll, die sich auch mit der Frage - von Werner Wolf zu Recht als ,vexed problem“ ${ }^{125}$ bezeichnet - nach der Narrativität von Instrumentalmusik

${ }^{123}$ Vgl. dazu z. B. Wolf: Narrativity in Instrumental Music?, S. 482; Ders: Transmedial Narratology: Theoretical Foundations and Some Applications (Fiction, Single Pictures, Instrumental Music). In: Narrative 25 (2017) H. 3, S. 256-285; Nünning, Vera u. Ansgar Nünning: Dies.: Produktive Grenzüberschreitungen: Transgenerische, intermediale und interdisziplinäre Ansätze in der Erzähltheorie. In: Dies. (Hrsg.): Erzähltheorie transgenerisch, intermedial, interdisziplinär. Trier: WVT 2002, S. 1-22; Ryan, Marie-Laure (Hrsg.): Narrative Across Media. The Languages of Storytelling. Lincoln/London: University of Nebraska Press 2004; Dies.: On the Theoretical Foundation of Transmedial Narratology. In: Meister, Jan Christoph (Hrsg., in Zusammenarbeit mit Tom Kindt u. Wilhelm Schernus): Narratology beyond Literary Criticism. Mediality, Disciplinarity. Berlin/New York: de Gruyter 2005, S. 1-23.

${ }^{124}$ Siehe Rajewsky: Intermedialität, S. 181.

${ }^{125}$ Wolf: Narrativity in Instrumental Music?, S. 480. 
verknüpft, da der Gegenstand der Studie auch rein instrumentale Kompositionen bilden. Das Narrative muss in diesem Fall im weiteren Sinne aufgefasst werden, was eine Abstrahierung voraussetzt: ${ }^{126}$ Folglich soll eine Perspektive eingenommen werden, die - so Rajewsky - ${ }^{127}$

jenseits bzw. über den einzelnen Medien verankert ist, um von diesem Blickpunkt aus unterschiedliche Manifestationsformen des i. w. S. Narrativen und damit zugleich modalen Gemeinsamkeiten und Differenzen verschiedener Genera Rechnung zu tragen, ohne dabei ein bestimmtes Medium, eine bestimmte Gattung oder einen bestimmten Erzählmodus zu privilegieren und zu einer Fundierungskategorie ,des Narrativen` zu erheben.

Eine transmediale Narratologie, die ,metonymisch gesprochen“128 von Genettes modaler Narratologie ausgeht, ${ }^{129}$ hält auch diese Studie in Anlehnung an Rajewsky für eine widersprüchliche Analysebasis: Es besteht die Gefahr eines - so Marie-Laure Ryan, die doch als eine der ersten Forscherinnen für eine transmediale Narratologie plädiert - ,,indiscriminating transfer of concepts“, 130 die ursprünglich für die Erforschung fiktionaler literarischer Texte konzipiert wurden. ${ }^{131}$

In einigen Aufsätzen zur Narrativität von Instrumentalmusik listet Wolf mithilfe eines prototypisch-kognitiven Ansatzes Stimuli instrumentaler Musikwerke, die Narrativität induzieren, auf. Diese Auflistung beruht auf der Feststellung obligatorischer und fakultativer Narrateme prototypischen Erzählens, wie z. B. Zeit, Raum, Figuren und Erzählinstanz, die beweisen, dass Narrativität ein graduierbares Phänomen ist, und der Erfassung von Narrativität in verschiedenen

\footnotetext{
${ }^{126}$ Siehe Chatman, Seymour: Coming to Terms. The Rhetoric of Narrative in Fiction and Film. Ithaca/London: Cornell University Press 1993, S. 117.

${ }^{127}$ Rajewsky, I. O.: Von Erzählern, die (nichts) vermitteln, S. 34, Herv. i. O.

${ }^{128}$ Ebd.

${ }^{129}$ Siehe Genette, Gérard: Die Erzählung. München: Fink 1994, S. 200 f.

${ }^{130}$ Ryan: Narrative Across Media, S. 34. Vgl. Auch Rajewsky, I.: Percorsi transmediali. Appunti sul potenziale euristico della transmedialità nel campo delle letterature comparate. In: Between 8 (November 2018) H. 16, S. 1- 24.

${ }^{131}$ Siehe auch Rajewsky: Percorsi transmediali, S. 18. Des Weiteren hebt u. a. Meister hervor, dass, obwohl seine Kategorien und Begriffe schnell zur narratologischen lingua franca geworden sind, Genette in seinen Texten keinen Anspruch erhebt, eine komplett kohärente und umfassende Narrativitätstheorie zu entwerfen, da er zudem nur Marcel Prousts À la recherche du temps perdu analysiert. Meister, Jan Christoph: Narratology. In: Interdisciplinary Center for Narratology (Hrsg.): the living handbook of narratology. 2011.<https:// www.lhn.unihamburg.de/node/48.html > (letzter Zugriff: 21.08.2020), Abschnitt 3.3.
} 
Medien und Gattungen dienen. Dieser Ansatz ermöglicht jedoch keine komplett „,medienübergreifende[] ,Draufsicht““.:132 Einerseits wird als Beispiel für eine prototypische Form des Erzählens eine Erzählform genannt, nämlich das Märchen, die das Primat verbalen Erzählens wieder betont, andererseits wirft der implizite Zusammenhang von Prototypen und Normalitätskonstruktionen Fragen zur terminologischen Adäquatheit auf. Zurecht unterstreicht allerdings Schmidt, dass sich der theoretische Rahmen der kognitiven Forschung mit ihrer Berücksichtigung der Wirkung auf die Rezipient*innen für die Beantwortung der Frage nach der Narrativität von Instrumentalmusik gut eignen könnte. ${ }^{133}$ Großes Verdienst von Wolfs Aufsätzen ist zudem, dass dort ein methodologischer Ansatz zur Identifikation von Elementen, die Narrativität hervorrufen, skizziert wird. Dies kann sowohl durch kompositionsexterne Stimuli wie kulturellen und historischen Kontext und Tendenzen im Gesamtwerk bestimmter Komponist*innen als auch durch kompositionsinterne Stimuli wie Paratexte geschehen: Auch die vorliegende Studie folgt bei der Analyse von Instrumentalkompositionen dieser Methode und schließt sich somit der Auffassung an, dass Instrumentalmusik eher als ,,narrative-inducing “134 zu betrachten sei. Die Bezugnahme auf Passagen und Figuren aus Thomas Manns Roman erleichtert dann auch in den hier betrachteten instrumentalen Kompositionen die Identifikation von Momenten, die Narrativität induzieren. Nicht nur unter dem Aspekt der Narrativität lässt sich Instrumentalmusik analysieren: Vielmehr ist die Deskriptivität in vielen Fällen - u. a. auf die Affinität von Musik und Lyrik zurückzuführen $-{ }^{135}$ heuristisch produktiv.

\subsubsection{Mediengrenze}

Eine letzte, nötige Präzisierung betrifft den für die Erforschung von Intermedialität zentralen Begriff der ,Mediengrenze‘. Dieser wurde in diesem Kapitel auch für die Beschreibung von Thomas Manns Roman verwendet; zunächst wird hier

\footnotetext{
${ }^{132}$ Rajewsky: Von Erzählern, die (nichts) vermitteln, S. 34.

${ }^{133}$ Vgl. Schmidt, Matthias: Literatur in (Instrumental-)musik. In: Gess, Nicola u. Honold, Alexander (Hrsg.): Handbuch Literatur \& Musik. Berlin/Boston: de Gruyter 2017, S. 114128, hier S. 115.

${ }^{134}$ Wolf: Narrativity in Instrumental Music?, S. 496, Herv. i. O. Die Übersetzung dieses Konzeptes ins Deutsche als ,Narrationsinduktion/Narrativitätsinduktion“ verdankt die Verfasserin Vorträgen und Erläuterungen von Werner Wolf im Rahmen der Summer School 2019 „Konstanten und Varianten des Erzählens in transgenerischen und transmedialen Kontexten“ (Saint-Hippolyte, 06.-10.08.2019).
}

${ }^{135}$ Schmidt: Literatur in (Instrumental-)musik, S. 119. 
auf ein Zitat aus Manns Entstehung des „Doktor Faustus“ verwiesen, in dem er das Wesen und die Aufgabe der Kunst folgendermaßen beschreibt:

[W]as sonst wäre es uns jemals zu tun, als unser Äußerstes zu geben? Alle Kunst, die den Namen verdient, zeugt von diesem Willen zum Letzten, dieser Entschlossenheit an die Grenze zu gehen, trägt das Signum, die Wundmale des ,utmost“. (Ent: 123)

Kunst zu produzieren heiße den Weg bis zu ihrer Grenze zu wagen; zugleich sei dieser Versuch nicht völlig harmlos und schließe eine gewisse Verletzbarkeit ein. Thomas Mann nimmt hier auf Kunstgrenzen Bezug, bedient sich aber gleichermaßen der Metapher der ,Grenze', ebenfalls in Verbindung mit künstlerischen Artefakten. Rajewsky kommt bezüglich der Funktion dieses Konzeptes zu folgender Aussage: ${ }^{136}$

Damit geht der Versuch einher, ein Umdenken hinsichtlich des Konzepts der Grenze und der Setzung von Differenzen zu befördern und von deren Auffassung im Sinne statischer Taxonomien, wie dies topisch mit dem Strukturalismus verbunden wird, zum dynamischen Potenzial der Grenzen selbst zu gelangen.

Auch die vorliegende Studie vertritt diese Auffassung von Mediengrenze und konzentriert sich auf den dynamischen, produktiven Austausch zwischen den Feldern der Literatur und der Musik sowie auf die zirkulären Verweisstrukturen, die dadurch entstehen. Eine Nivellierung von Mediendifferenzen und Gattungskonventionen würde die ,Möglichkeiten und Freiräume“, ${ }^{137}$,,die sich für die künstlerische bzw. kulturelle Praxis durch konventionelle Setzungen auf verschiedensten Ebenen ergeben“, 138 kaum beobachtbar machen. Die Betrachtung von Grenzen als „Ermöglichungsstrukturen, als Spielräume“, ${ }^{39}$ scheint besonders bei der Analyse von Kompositionen der Neuen Musik ergiebig, insofern sie oft auf Experimentieren beruhen. Um einige Beispiele aus dieser Untersuchung zu nennen: Lars Petter Hagen experimentiert in To Zeitblom mit der Grenze zwischen Volksmusik und klassischer Musik sowie zwischen theatralischen und musikalischen Konventionen und Konrad Boehmer kombiniert ebenfalls populäre Musikgenres mit elektronischer Musik. Tatsächliche Grenzen existieren nicht, es handelt sich um ein aus heuristischen Gründen eingeführtes Analysekonzept, das

\footnotetext{
${ }^{136}$ Rajewsky: Von Erzählern, die (nichts) vermitteln, S. 38.

${ }^{137}$ Ebd., S. 63.

${ }^{138}$ Ebd.

${ }^{139}$ Ebd.
} 
u. a. historische Kontextualisierungen und einen verschärften Blick auf den Raum des Dazwischen fördert. ${ }^{140}$

\subsection{Quellen}

Die Analyse der Kompositionen stützt sich auf Partituren, Aufnahmen und Inszenierungen, um allen Dimensionen der jeweiligen musikalischen Form gerecht $\mathrm{zu}$ werden, welche auch die Besonderheit des Mediums selbst ausmachen und die Notwendigkeit eines kommunikativ-semiotischen Deutungsparadigmas noch deutlicher herausstellen. Im Fall von Medienkombinationen ist es außerdem gerade die Kopplung dieser Medien, die zu untersuchen sind, insofern sie der Transposition des literarischen Textes auch durch die Inszenierung neue Konnotationen verleihen kann. ${ }^{141}$ Selbstkommentare, Selbstaussagen von Autor*innen sowie biographische Informationen zu ihnen (dies betrifft auch den ersten Teil jedes Kapitels zum Roman) dienen lediglich der Kontextualisierung; generell folgt die vorliegende Studie diesbezüglich dem von Stefan Börnchen in seiner Monographie vorgeschlagenen und umgesetzten Prozedere Manns Selbstaussagen gegenüber, das er - Bezug nehmend auf Paul de Man - wie folgt beschreibt: ${ }^{142}$

Eine Interpretation kann sich auf Selbstkommentare des Autors Thomas Manns stützen, muß aber diese wie alle anderen Texte interpretieren: Denn in den - wie gesehen: widersprüchlichen - Selbstaussagen tritt nicht die Absicht des Autors klar und eindeutig zu Tage. In Hinsicht auf Selbstkommentare gilt nach wie vor Paul de Mans Wort, daß „,die einzige irreduzible ,Intention“ eines Textes die seiner Konstituierung ist".

Neben der Präzisierung, wie die Untersuchung mit den Selbstaussagen Thomas Manns umgeht, scheint hier eine zweite Präzisierung wichtig. Den Leser*innen

\footnotetext{
${ }^{140}$ Siehe dazu auch Backe, Hans-Joachim: Medialität und Gattung. In: Zymner, Rüdiger (Hrsg.): Handbuch Gattungstheorie. Stuttgart: Metzler 2010, S. 105 ff., hier: S. 105; Sombroek, Andreas: Eine Poetik des Dazwischen. Zur Intermedialität und Intertextualität bei Alexander Kluge. Bielefeld: transcript 2005; Elleström, Lars (Hrsg.): Media Borders, Multimodality and Intermediality. Basingstoke (u. a.): Palgrave Macmillan 2010 (insb. Part II u. Part V).

${ }^{141}$ Siehe auch Stollberg: Kombination von Literatur und Musik, S. 71.

${ }^{142}$ Börnchen: Kryptenhall, S. 72 (Zitat aus: de Man, Paul: Lesen (Proust). In: Ders.: Allegorien des Lesens. Frankfurt am Main: Suhrkamp 1988 [New Haven 1979], S. 91-117, hier: S. 98).
} 
wird sicherlich auffallen, dass die Analysen wiederholt auf Adornos Musikphilosophie rekurrieren, was eine Konstante der meisten Publikationen zu Doktor Faustus darstellt. In der Forschungsliteratur zum Roman wird nicht selten anhand biographischer Dokumente unterschiedlicher Art der Frage nachgegangen, welche Rolle denn Adorno bei der Entstehung von Thomas Manns Roman gespielt habe. So unterstreicht Bodo Heimann beispielsweise den bedeutenden Einfluss Adornos und zitiert aus einem Brief Schönbergs, in dem er den Philosophen als echten Kenner des von ihm entwickelten musikalischen Systems und Thomas Mann als Laien bezeichnet. ${ }^{143}$ In einem vorher erschienenen Aufsatz (1993) vertritt auch Claudia Albert die gleiche Auffassung und kommt zum Schluss, dass „die auf das Teufelsgespräch folgenden Kompositionen Leverkühns, insbesondere also die ,Apocalipsis cum figuris ' und die Kantate ,Dr. Fausti Weheklag' als imaginäre Werke Adornos betrachtet werden"144 müssten. Ganz anderer Auffassung ist hingegen Matthias Schmidt: Er hebt einerseits die Einflüsse von Kreneks Music Here and Now hervor, um die Vielfalt von Thomas Manns konsultierten Quellen gegen die alleinige Reduzierung auf die Musikphilosophie Adornos deutlich zu machen, andererseits zeigt er anhand anderer autobiographischer Dokumente, dass sich Schönbergs Ressentiment nicht gegen Mann, sondern gegen Adorno richtete. ${ }^{145}$ Dass sich diese Kontroverse nicht lösen lässt, auch weil sie zutiefst in einem hermeneutischen Interpretationsparadigma verankert ist, mag nun bereits deutlich geworden sein. Diese Studie zielt nicht darauf ab, Adornos Einfluss auf die Entstehung von Doktor Faustus zu rekonstruieren. Vielmehr möchte sie in ihrem intermedial angelegten Prozedere Bezüge beleuchten, die sowohl im Roman als auch in den Kompositionen eine Rolle spielen. Da Adornos Auffassungen im Text und in den Musikwerken mehrfach zum Tragen kommen, versteht es sich von selbst, dass diese Arbeit wiederholt auf sie verweist. Dies geht nicht aus der Absicht einer Privilegierung dieser Musikphilosophie vor anderen, sondern aus der beobachteten Sonderstellung in den Kompositionen hervor. Als Paradebeispiel dient hierfür Hagens Stück To Zeitblom mit seiner Parodie von Adornos

\footnotetext{
${ }^{143}$ Siehe Heimann, Bodo: Thomas Manns ,Doktor Faustus‘ und die Musikphilosophie Adornos. In: Ders. (Hrsg.): Literatur und Freiheit von Lessing bis zur Gegenwart. Frankfurt am Main: Lang 2014, S. 211-228 (zu Schönbergs Brief siehe insb. S. 212).

${ }^{144}$ Albert, Claudia: „Doktor Faustus“: Schwierigkeiten mit dem strengen Satz und Verfehlung des Bösen. In: Heinrich Mann Jahrbuch 11 (1993), S. 99-111, hier: S. 103.

${ }^{145}$ Vgl. Schmidt, Matthias: „Unangreifbar nur die Gestalt“. Thomas Mann - Ernst Krenek - Theodor W. Adorno und die Musik im „Doktor Faustus“. In: Henke, Matthias (Hrsg.): Schönheit und Verfall: Beziehungen zwischen Thomas Mann und Ernst Krenek - (mehr als) ein Tagungsbericht (Thomas-Mann-Studien Bd. 47). Frankfurt am Main: Klostermann 2015, S. 135-153 (zu Schönbergs Quellen siehe insb. S. 143).
} 
theoretischen Postulaten inmitten der Aufführung. Im Prozess der Rekonstruktion der kompositorischen Rezeptionsgeschichte von Doktor Faustus scheint folglich erforderlich, auf einige Schriften Adornos einzugehen.

\subsection{Aufbau der Arbeit}

Diese Arbeit ist so strukturiert, dass jedes Kapitel aus zwei Hauptteilen besteht: Der erste Teil jeweils widmet sich Leverkühns fiktiven Werken, bestimmten Romanstellen oder Figuren. Zugleich wird eine (werkinterne) intermediale Analyse durchgeführt oder es werden hauptsächlich intradiegetische Motive und Diskurse näher beleuchtet. Den Kapitelüberschriften lässt sich entnehmen, was im Zentrum der Untersuchung steht. Der zweite Hauptteil jeweils wagt den Sprung zur werkexternen und oft auch zur harten Intermedialität und befasst sich mit Kompositionen, die Leverkühns fiktive Werke nachvertonen oder sich mit bestimmten Romanpassagen und -figuren auseinandersetzen. Hier wird vor allem untersucht, ob die vorher beleuchteten Motive und Diskurse in der Komposition wiederzufinden sind, und wenn ja, wie sie im neuen Medium dargestellt werden. Falls diese in der Komposition nicht vorliegen oder nur eine marginale Rolle spielen, dann wäre diese Feststellung für die Analyse durchaus von Bedeutung, denn sie kann zur Beobachtung führen, dass solche Motive und Diskurse im jeweiligen Musikwerk durch andere ersetzt werden, oder dass Motive und Diskurse, die vorher nicht analysiert wurden, weil sie vielleicht keine zentrale Stellung im Roman einnehmen, doch in der Komposition einen Ehrenplatz bekommen und somit verstärkt werden. All diese Beobachtungen sind symptomatisch für die Rezeption des Romans im jeweiligen Werk, der es als oberstes Ziel schließlich nachzugehen gilt: Jede intermediale Transposition bzw. Bezugnahme schreibt Thomas Manns Roman mit den eigenen Mitteln neu und fordert gleichzeitig zum aktiven Lesen und Hören auf. ${ }^{146}$

${ }^{146}$ Siehe Wirth: Hypertextuelle Aufpfropfung, S. 23; Barthes, R.: Die Lust am Text. Übers. v. Traugott König. Frankfurt am Main: Suhrkamp 1974 [Paris 1973], S. 68; Ders.: S/Z. Übers. v. Jürgen Hoch. Frankfurt am Main: Suhrkamp 1987 [Paris 1970], S. 8 ff. 
Open Access Dieses Kapitel wird unter der Creative Commons Namensnennung - Nicht kommerziell - Keine Bearbeitung 4.0 International Lizenz (http://creativecommons.org/lic enses/by-nc-nd/4.0/deed.de) veröffentlicht, welche die nicht-kommerzielle Nutzung, Vervielfältigung, Verbreitung und Wiedergabe in jeglichem Medium und Format erlaubt, sofern Sie den/die ursprünglichen Autor(en) und die Quelle ordnungsgemäß nennen, einen Link zur Creative Commons Lizenz beifügen und angeben, ob Änderungen vorgenommen wurden. Die Lizenz gibt Ihnen nicht das Recht, bearbeitete oder sonst wie umgestaltete Fassungen dieses Werkes zu verbreiten oder öffentlich wiederzugeben.

Die in diesem Kapitel enthaltenen Bilder und sonstiges Drittmaterial unterliegen ebenfalls der genannten Creative Commons Lizenz, sofern sich aus der Abbildungslegende nichts anderes ergibt. Sofern das betreffende Material nicht unter der genannten Creative Commons Lizenz steht und die betreffende Handlung nicht nach gesetzlichen Vorschriften erlaubt ist, ist auch für die oben aufgeführten nicht-kommerziellen Weiterverwendungen des Materials die Einwilligung des jeweiligen Rechteinhabers einzuholen.

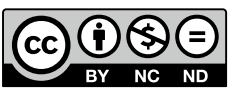

